\title{
Détection d'amines non volatiles dans quelques fromages
}

\author{
par
}

\author{
A. DE VUYST, W. VERVACK et M. FOULON \\ (avec la collaboration de Claudette SACRE)
}

\section{INTRODUCTION}

J. Lederer [1] attirait l'attention sur une certaine incompatibilité entre le fromage et des médicaments antidépressifs.

Cette incompatibilité est appelée "Cheese Syndrom » par un médecin anglais, Maan [2]. Il signalait le cas d'une de ses patientes qui, traitée par un antidépressif, présentait de l'agitation et des maux de tête chaque fois qu'elle mangeait du fromage.

CoOper, Magnus et Rose [3] ont analysé les causes des intolérances survenues chez des patients traités par un antidépressif : 20 p. 100 des patients firent des crises d'intolérance et $1 / 3$ des sujets atteints, avait consommé du fromage 1 à $3 \mathrm{~h}$ avant la crise. Blackwell et Marley [4] purent mettre en évidence en 1964 la relation qui existe entre l'intolérance aux médicaments antidépressifs et la consommation de certains fromages.

Les amines primaires non volatiles se forment à partir des acides aminés de la protéine hydrolysée au cours de la maturation du fromage. Cette formation d'amines se fait par des réactions de décarboxylation aux dépens des acides aminés avec formation d'amines et de $\mathrm{CO}_{2}$.

La tyramine provient de la tyrosine, l'histamine de l'histidine, la cadavérine de la lysine et la putrescine de l'arginine avec un stade intermédiaire, l'ornithine.

Chez le sujet normal, les amines sont dégradées, au niveau du foie, par la mono-amine-oxydase (M.A.O.).

Travail réalisé au Centre de Recherches Zootechniques de l'Université de Louvain (Directeur : Professeur Dr A. De Vuyst) B - 3042 Lovenjoel (Belgique). 
C'est ainsi que, d'après Lederer J. [1] on aura, pour la tyrosine le schéma suivant :

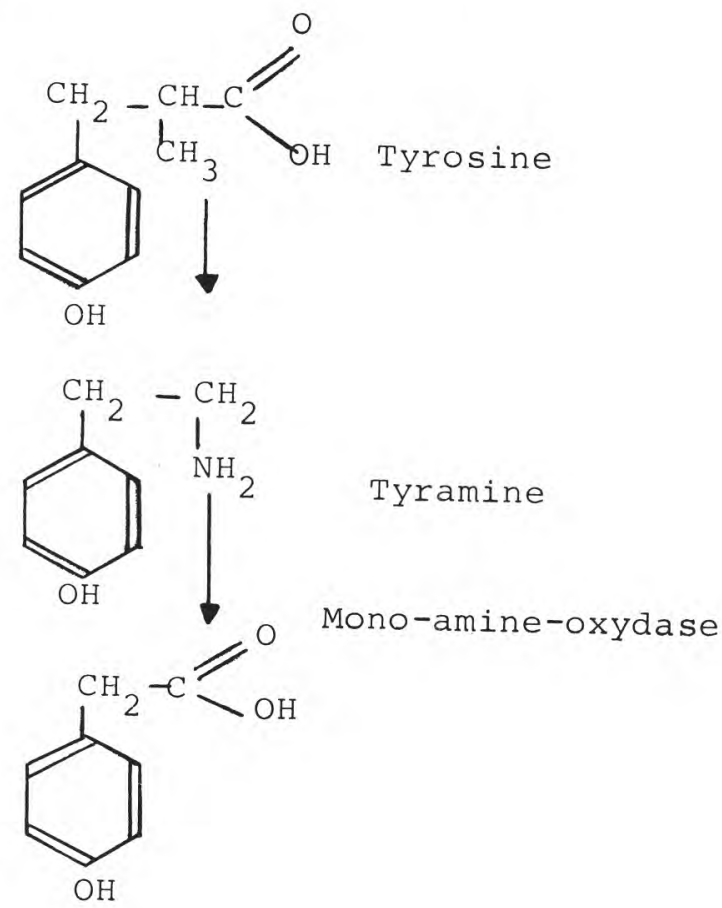

Normalement, le produit de dégradation est éliminé.

Chez le sujet qui reçoit un inhibiteur de la M.A.O., la tyramine passe dans le sang et provoque une élévation de la tension artérielle, élévation d'autant plus forte que l'aliment consommé est plus riche en tyramine, ce qui est le cas des fromages " mûrs ", fortement affinés.

Ce « Cheese Syndrom » peut parfois prendre une allure tragique et laisser des séquelles plus ou moins graves.

C'est pourquoi nous avons jugé intéressant de déterminer la présence de certaines amines primaires non volatiles dans différents types de fromages consommés couramment en Belgique.

Les amines dosées sont : l'histamine, la tyramine, la putrescine et la cadavérine.

Pour déceler et doser ces amines, nous utilisons une méthode de dosage sur colonne d'échangeur d'ions dérivant de la méthode de 
dosage des acides aminés de Stein et Moore. Cette méthode fut mise au point dans notre laboratoire [5].

Toutefois, pour que la séparation entre les différentes amines puisse se faire avec efficacité, il est nécessaire de prévoir un volume mort d'environ $10 \mathrm{ml}$ au-dessus de la surface de résine, afin que le changement de tampon puisse s'effectuer non pas de manière brusque, mais sous forme de gradient.

Les déterminations se font au moyen d'un analyseur automatique d'acides aminés, Beckman Unichrom.

\section{RESULTATS ANALYTIQUES}

Pour chaque variété de fromage, nous avons analysé quatre échantillons, afin de pouvoir établir des comparaisons entre les différents résultats.

Les résultats obtenus sont repris dans les tableaux 1 et 2 .

\section{TABLEAU 1}

Teneurs en amines de quelques fromages en mg d'amines par gramme de fromage

\begin{tabular}{|c|c|c|c|c|}
\hline & Histamine & Tyramine & Putrescine & Cadaverine \\
\hline Brie & $\begin{array}{c}\text { traces } \\
0,023 \\
0,014 \\
\text { traces }\end{array}$ & $\begin{array}{c}0,008 \\
0,091 \\
0,398 \\
\text { traces }\end{array}$ & $\begin{array}{c}0,020 \\
0,072 \\
0,122 \\
\text { traces }\end{array}$ & $\begin{array}{c}\text { traces } \\
0,473 \\
0,553 \\
\text { traces }\end{array}$ \\
\hline Camembert & $\begin{array}{c}0,024 \\
\text { traces } \\
\text { traces } \\
\text { traces } \\
0,139\end{array}$ & $\begin{array}{c}\text { traces } \\
0,047 \\
0,020 \\
\text { traces } \\
0,660\end{array}$ & $\begin{array}{c}\text { traces } \\
0,009 \\
0,013 \\
\text { traces } \\
0,605\end{array}$ & $\begin{array}{c}\text { traces } \\
0,026 \\
0,019 \\
\text { traces } \\
1,180\end{array}$ \\
\hline Herve & $\begin{array}{l}0,014 \\
0,083 \\
0,036 \\
0,031\end{array}$ & $\begin{array}{l}0,077 \\
0,239 \\
0,137 \\
0,051\end{array}$ & $\begin{array}{l}0,077 \\
0,446 \\
0,427 \\
0,060\end{array}$ & $\begin{array}{l}0,169 \\
0,772 \\
1,320 \\
0,285\end{array}$ \\
\hline Bruxelles & $\begin{array}{l}0,090 \\
0,031 \\
0,006 \\
0,025\end{array}$ & $\begin{array}{l}0,231 \\
0,129 \\
0,129 \\
0,191\end{array}$ & $\begin{array}{l}0,109 \\
0,051 \\
0,040 \\
0,048\end{array}$ & $\begin{array}{l}0,124 \\
0,030 \\
0,107 \\
0,016\end{array}$ \\
\hline
\end{tabular}


TABLEAU 1 (suite)

\begin{tabular}{|c|c|c|c|c|}
\hline & Histamine & Tyramine & Putrescine & Cadaverine \\
\hline Roquefort & $\begin{array}{c}0,024 \\
0,002 \\
0,049 \\
\text { traces }\end{array}$ & $\begin{array}{l}0,023 \\
0,007 \\
0,014 \\
0,117\end{array}$ & $\begin{array}{c}0,020 \\
\text { traces } \\
0,131 \\
0,109\end{array}$ & $\begin{array}{c}0,214 \\
\text { traces } \\
0,303 \\
0,114\end{array}$ \\
\hline Bleu de Bresse & $\begin{array}{c}4,093 \\
3,283 \\
\text { traces } \\
\text { traces }\end{array}$ & $\begin{array}{c}0,313 \\
0,107 \\
\text { traces } \\
\text { traces }\end{array}$ & $\begin{array}{c}0,013 \\
0,151 \\
\text { traces } \\
\text { traces }\end{array}$ & $\begin{array}{c}0,195 \\
2,271 \\
\text { traces } \\
\text { traces }\end{array}$ \\
\hline Edam & $\begin{array}{c}0,088 \\
\text { traces } \\
\text { traces } \\
\text { traces }\end{array}$ & $\begin{array}{c}\text { traces } \\
\text { traces } \\
\text { traces } \\
0,197\end{array}$ & $\begin{array}{l}\text { traces } \\
\text { traces } \\
\text { traces } \\
\text { traces }\end{array}$ & $\begin{array}{l}\text { traces } \\
\text { traces } \\
\text { traces } \\
\text { traces }\end{array}$ \\
\hline Maredsous & $\begin{array}{c}0,231 \\
\text { traces } \\
\text { traces } \\
\text { traces }\end{array}$ & $\begin{array}{c}0,017 \\
\text { traces } \\
\text { traces } \\
0,013\end{array}$ & $\begin{array}{c}\text { traces } \\
\text { traces } \\
\text { traces } \\
0,004\end{array}$ & $\begin{array}{c}0,068 \\
\text { traces } \\
\text { traces } \\
0,010\end{array}$ \\
\hline Gruyère & $\begin{array}{c}0,088 \\
\text { traces } \\
\text { traces } \\
0,200\end{array}$ & $\begin{array}{l}0,067 \\
0,062 \\
0,065 \\
0,201\end{array}$ & $\begin{array}{c}0,160 \\
0,018 \\
\text { traces } \\
0,029\end{array}$ & $\begin{array}{c}\text { traces } \\
0,057 \\
\text { traces } \\
0,020\end{array}$ \\
\hline Cheddar & $\begin{array}{l}0,265 \\
0,046 \\
0,037 \\
0,272\end{array}$ & $\begin{array}{l}1,085 \\
0,650 \\
0,353 \\
0,648\end{array}$ & $\begin{array}{l}0,996 \\
0,387 \\
0,447 \\
0,680\end{array}$ & $\begin{array}{l}0,408 \\
0,166 \\
0,016 \\
0,120\end{array}$ \\
\hline
\end{tabular}

TABLEAU 2

Teneurs minimales et maximales en amines de quelques fromages en mg d'amines par gramme de fromage

\begin{tabular}{l|l|l|l|l|l|l|l|l}
\hline & \multicolumn{2}{|c|}{ Histamine } & \multicolumn{2}{|c|}{ Tyramine } & \multicolumn{2}{c|}{ Putrescine } & \multicolumn{2}{c}{ Cadaverine } \\
\cline { 3 - 5 } & Min. & Max. & Min. & Max. & Min. & Max. & Min. & Max. \\
\hline & & & & & & & & \\
Brie & traces & 0,023 & traces & 0,398 & traces & 0,122 & traces & 0,553 \\
Camembert & traces & 0,139 & traces & 0,660 & traces & 0,605 & traces & 1,180 \\
\hline
\end{tabular}


TABLEAU 2 (suite)

\begin{tabular}{|c|c|c|c|c|c|c|c|c|}
\hline & \multicolumn{2}{|c|}{ Histamine } & \multicolumn{2}{|c|}{ Tyramine } & \multicolumn{2}{|c|}{ Putrescine } & \multicolumn{2}{|c|}{ Cadaverine } \\
\hline & Min. & Max. & Min. & Max. & Min. & Max. & Min. & Max. \\
\hline Herve & 0,014 & 0,083 & 0,051 & 0,239 & 0,060 & 0,446 & 0,169 & 1,320 \\
\hline Bruxelles & 0,006 & 0,090 & 0,129 & 0,231 & 0,040 & 0,109 & 0,016 & 0,124 \\
\hline Roquefort & traces & 0,049 & 0,007 & 0,117 & traces & 0,131 & traces & 0,303 \\
\hline Bleu de Bresse & traces & 4,093 & traces & 0,313 & traces & 0,151 & traces & 2,271 \\
\hline Edam & traces & 0,088 & traces & 0,197 & traces & traces & traces & traces \\
\hline Maredsous & traces & 0,231 & traces & 0,017 & traces & 0,004 & traces & 0,068 \\
\hline Gruyère & traces & 0,200 & 0,062 & 0,201 & traces & 0,160 & traces & 0,057 \\
\hline Cheddar & 0,037 & 0,272 & 0,353 & 1,085 & 0,387 & 0,996 & 0,016 & 0,408 \\
\hline
\end{tabular}

N.B. : Dans ces tableaux, il a été convenu d'appeler traces, des teneurs en amines inférieures à $0,002 \mathrm{mg}$ par gramme de fromage, car en-dessous de cette valeur, les résultats ne sont plus calculables.

\section{DISCUSSION DES RESULTATS}

Nous avons testé la méthode analytique utilisée en comparant les résultats de dosages d'amines dans des fromages additionnés ou non de quantités connues d'amines. Les résultats obtenus montraient des rendements de 81 à 99 p. 100 des amines ajoutées.

La méthode de dosage s'avérait donc très valable, d'autant plus que les erreurs sur les dosages des amines contenues dans un fromage sont faibles vis-à-vis des variations dans les teneurs en amines pour un même groupe de fromage.

Nous avons comparé nos données avec celles obtenues par différents auteurs. 
Spettoli, en 1971 [6], trouve les résultats suivants exprimés en mg d'amines par gramme de fromage.

\begin{tabular}{l|l|c|c|c}
\hline \multicolumn{1}{c|}{ Fromage } & \multicolumn{1}{c|}{ Histamine } & Tyramine & Putrescine & Cadaverine \\
\cline { 2 - 3 } Cheddar & 0,050 & $0 \longrightarrow 0,953$ & - & - \\
Camembert & $0,209 \rightarrow 0,255$ & $0,090 \rightarrow 2,000$ & $0,092 \rightarrow 0,143$ & - \\
Edam & $0,065 \rightarrow 0,240$ & $0 \longrightarrow 0,050$ & 0 & - \\
\hline
\end{tabular}

Kosikowsky et Dahlberg, en 1948 [7], déterminent la tyramine dans les fromages suivants, exprimée en $\mathrm{mg}$ de tyramine par gramme de fromage.

\begin{tabular}{l|c}
\hline \multicolumn{1}{c|}{ Fromage } & Tyramine \\
Edam & $0,100 \longrightarrow 0,214$ \\
Roquefort & 0,049 \\
Bleu & 0,266 \\
Camembert & 0,125 \\
\hline
\end{tabular}

En comparant ces tableaux entre eux ou avec nos propres données, on doit admettre que les résultats sont tout à fait discordants.

Ces variations doivent être mises sur le compte des conditions de maturation des fromages.

Nous n'en voulons pour preuve que l'exemple suivant :

- pour le Bleu de Bresse, nous avons analysé deux marques différentes et cela deux fois chacune. Nous avons obtenu les données suivantes (exprimées en $\mathrm{mg}$ d'amines par gramme de fromage). 


\begin{tabular}{|c|c|c|c|c|c|}
\hline \multicolumn{2}{|c|}{ Marque $\mathrm{n}^{\circ}$} & Histamine & Tyramine & Putrescine & Cadavérine \\
\hline \multirow[t]{2}{*}{ I } & a & 4,093 & 0,313 & 0,013 & 0,194 \\
\hline & b & 3,283 & 0,106 & 0,151 & 2,271 \\
\hline & a & traces & traces & traces & traces \\
\hline & b & traces & traces & traces & traces \\
\hline
\end{tabular}

Cet exemple est très significatif.

La teneur en amines des fromages est donc extrêmement variable. Cela semble dû pour la plus grande part, aux conditions de maturation et d'affinage qui dépendent de nombreux facteurs physiques, chimiques et bactériologiques.

Plus un fromage est mûr et plus il contiendra une quantité importante d'amines.

Kosikowsky et Dahlberg [8] montrèrent en 1948 la corrélation entre l'arôme et la teneur en tyramine dans le Cheddar. Selon ces auteurs, l'emploi de Streptococcus faecalis augmente l'arôme du Cheddar et la décarboxylation de la tyrosine en tyramine. Pour une même variété de fromage on peut avoir des variations dans les teneurs respectives en amines (tab. 1).

Les fromages à pâte molle ont des teneurs certaines en amines, ce qui résulte de leur type de maturation.

Les fromages à pâte dure montrent des variations : le Gruyère contient des amines alors que l'Edam et le Maredsous n'en contiennent pratiquement pas. Le Cheddar, dans ce groupe, contient une forte proportion d'amines.

\section{CONCLUSION}

Nous avons pu déterminer des quantités assez variables d'histamine, de tyramine, de putrescine et de cadavérine dans des fromages consommés en Belgique.

Les fromages à pâte molle contiennent des quantités plus ou moins grandes d'amines. Les fromages à pâte dure n'en contiennent que très peu, sauf le Cheddar qui, lui, contient beaucoup d'amines. 
La maturation joue un rôle de premier ordre dans la transformation des acides aminés en amines.

Les facteurs physiques (température, $\mathrm{pH}$...), chimiques (décarboxylation) et microbiologiques (Streptococcus faecalis) provoquent la formation d'amines.

Les amines contenues dans les fromages ne sont pas nuisibles pour l'homme normal. Elles peuvent être toxiques et présenter un réel danger dans certains états pathologiques qui nécessitent la prise d'inhibiteurs de la mono-amino-oxydase, par des malades recevant des médicaments antidépressifs.

Il serait intéressant de pouvoir suivre la formation et le développement des teneurs en amines dans un même fromage au cours des différentes étapes de sa maturation ou de sa conservation.

Cette idée nous vient du fait que dans certaines marques de fromage, nous avons trouvé zéro ou une quantité considérable d'amines non volatiles.

\section{Ré s u m é}

Dans le présent travail, les auteurs ont déterminé quantativement les amines primaires non volatiles de quelques fromages courants.

Ils trouvent des teneurs assez variables, suivant les types de fromages.

\section{S u m m a r y}

The authors have determinated quantitatively the concentration of non volatile primary amines in different type of cheeses.

They found very variable contents of amines in relation with the type of investigated cheeses.

Reçu pour publication en mars 1976.

\section{Bibliographie}

[1] LEDERER (J.). (1968). - Note de diététique pratique. Incompatibilité entre le fromage et certains antidépressifs. Louvain Méd., 87, 521-523.

[2] MaAn (S. A.) (1963). - Tranylcypromine and cheese. Lancet, II, 639.

[3] Cooper (A. J.), Magnus (R. V.) and Rose (M. J.) (1964). - A hypertensive syndrome with tranylcypromine medication. Lancet, I, 527-529. 
[4] Blackwell (B.) and Marley (E.) (1964). - Interaction between cheese and mono-amine-oxydase inhibitors in cats and rats. Lancet, I, 530-531.

[5] De Vuyst (A.), Vervack (W.), Vanbelle (M.) et Jadin (V.) (1969). — Le dosage des amines présentes dans l'ensilage par chromatographie sur colonnes échangeuses d'ions. Zeitschrift für Tierphysiologie, Tierernährung und Futtermittelkunde, 25, 350-357.

[6] Spettoli (P.) (1971). - Contenuto in amine biogene di 24 formaggi italiani. Industrie Agrarie, 9, 42-46.

[7] Kosikowski (F. V.) and DAHLBERg (A. C.) (1948). - The tyramine content of Cheese. Journal of Dairy Science, 31, 293-303.

[8] Kosikowski (F. V.) and Dahlberg (A. C.) (1948). - The relationship of the amounts of tyramine and the numbers of Streptoccocus faecalis on the intensity of flavor in American Cheddar Cheese. Journal of Dairy Science, 31, 305-314. 